\title{
Comparability of total cardiovascular disease risk estimates using laboratory and non-laboratory based assessments in urban-dwelling South Africans: The CRIBSA study
}

\author{
N Peer, ${ }^{1} \mathrm{MB}$ ChB, MBA, MPH, PhD; C Lombard, ${ }^{2} \mathrm{MSc}, \mathrm{PhD}$; K Steyn, ${ }^{3} \mathrm{NED}, \mathrm{MSc}, \mathrm{MD}$; T Gaziano, ${ }^{4} \mathrm{MSc}, \mathrm{MD}$; \\ N Levitt, ${ }^{3,5}$ MB ChB, MD, FCP (SA) \\ ${ }^{1}$ Non-communicable Diseases Research Unit, Medical Research Council, Durban, South Africa \\ ${ }^{2}$ Biostatistics Unit, Medical Research Council, Cape Town, South Africa \\ ${ }^{3}$ Chronic Disease Initiative for Africa, Department of Medicine, Faculty of Health Sciences, University of Cape Town, South Africa \\ ${ }^{4}$ Division of Cardiovascular Medicine, Brigham and Women's Hospital, Harvard Medical School, Boston, USA \\ ${ }^{5}$ Division of Diabetic Medicine and Endocrinology, Department of Medicine, Faculty of Health Sciences, University of Cape Town, South Africa
}

Corresponding author: N Peer (nasheeta.peer@mrc.ac.za)

Objectives. To establish the prevalence and determinants of the 10-year risk of a cardiovascular disease (CVD) event in 25 - 74-year-old black Africans in Cape Town, South Africa, using Framingham laboratory- and non-laboratory-based and National Health and Nutrition Examination Survey (NHANES) I non-laboratory-based equations.

Methods. CVD risk factors were determined by questionnaires, clinical measurements and biochemical analyses. Survey logistic regression analyses assessed the sociodemographic determinants of CVD risk $\geq 20 \%$.

Results. There were 1025 participants, 369 men and 656 women. Mean 10-year risk for a CVD event by Framingham laboratory- and non-laboratory-based and NHANES I non-laboratory-based equations for men was 9.0\% (95\% confidence interval 7.7 - 10.3 ), $11.1 \%$ (9.6 - 12.6) and 9.0\% (7.6 - 10.3), and for women 5.4\% (4.7 - 6.1), 6.8\% (5.9 - 7.7) and 8.7\% (7.6 - 9.8). Correlations between laboratory- and non-laboratory-based scores were high $(0.915$ - 0.963). The prevalence of laboratory-based CVD risk $\geq 20 \%$ was $13.0 \%$ in men and $6.1 \%$ in women. In the logistic model for men, $\leq 7$ years of education (odds ratio 3.09; 95\% CI 1.67 - 5.71) and being unemployed (3.44; 1.21 - 9.81) compared with employed were associated with laboratory-based high risk. In women, high risk was associated with $\leq 7$ years of education (4.20; 1.96 - 9.01), living in formal v. informal housing $(2.74 ; 1.24-6.06)$ and being poor (middle v. lowest tertile $0.29 ; 0.13$ - 0.66$)$. In the Framingham non-laboratory-based logistic models there were no changes in the direction or significance of the variables except for housing, which was no longer significant in women.

Conclusions. Comparability of laboratory- and non-laboratory-based CVD risk estimates illustrates the utility of the latter in resourceconstrained settings.

S Afr Med J 2014;104(10):691-696. DOI:10.7196/SAMJ.8125

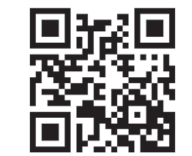

Treatment of cardiovascular disease (CVD) risk factors has traditionally been based on the presence or absence of a single CVD risk factor, such as hypertension, hyperlipidaemia or diabetes, without considering the continuous relationship between blood pressure (BP), blood glucose, blood cholesterol and cardiovascular risk. ${ }^{[1]}$ While this approach appears straightforward, it may result in committing some individuals with only a small cardiovascular risk to years of unnecessary treatment or, conversely, neglecting to treat individuals with an overall higher risk. ${ }^{[1,2]}$ This is because a combination of several slightly elevated risk factors may result in a much higher total risk than a single, more strikingly raised factor. $^{[3,4]}$

Moreover, single risk factor approaches are neither cost-effective nor affordable for poorer individuals and in developing regions with limited resources. ${ }^{[2]}$ Given the enormous burden of CVD and the high costs of management, it is therefore essential to prioritise cost-effective approaches that target high-risk individuals. Adoption of the multifactorial CVD risk assessment approach to identify high-risk individuals who need interventions is widely advocated, with the initiation of therapy based on the predicted absolute cardiovascular risk of the individual. ${ }^{[1]}$ The total CVD risk assessment approach is particularly recommended as a cost-effective strategy in developing regions with scarce resources. Effective CVD prevention therefore warrants a paradigm shift from the treatment of single risk factors in isolation, to the management of total CVD risk with an improvement in the profile of all risk factors that will lead to the development of CVD.

Several computerised methods for estimating total cardiovascular risk have been developed. The first, best known and most frequently used risk estimation system was developed by the Framingham Heart Study researchers in the USA. ${ }^{[3,4]}$ This score has been validated across different populations, modified for use in several countries and recommended by numerous international guideline committees for CVD prevention.

In South Africa (SA), the South African Heart Association and the Lipid and Atherosclerosis Society of Southern Africa, ${ }^{[5]}$ as well as the Southern African Hypertension Society in conjunction with the National Department of Health $(\mathrm{DoH}),{ }^{[6]}$ have adopted a cardiovascular risk stratification approach for the management of CVD risk factors. Given the lack of prospective data in the African setting and the absence of locally validated total CVD risk assessment tools, and in view of the fact that the Framingham risk scores have been validated in white and black populations and are transportable to other culturally diverse populations, this approach has been considered appropriate for local use. ${ }^{[5]}$ 
Most scoring systems require expensive laboratory tests; however, recent advances include the development of models that use simple office-based predictors easily obtained in primary care and do not require laboratory testing. The body mass index (BMI) has replaced total cholesterol (TC) and high-density lipoprotein (HDL) cholesterol in these models. Considering the increasing burden of CVD and the need for cost-effective use of limited resources in the developing world, the introduction of non-laboratory-based tools in this milieu is highly relevant. The use of non-laboratory-based total CVD risk assessment has the potential to improve worldwide utility of the risk scores and enhance targeted CVD prevention efforts, ${ }^{[3,4]}$ particularly in resource-constrained settings such as SA, where widespread laboratory availability is problematic and not economically feasible.

This study aimed to determine the 10 -year risk of developing a CVD event in the black population of Cape Town using the Framingham laboratory- and non-laboratory-based equations. Additionally, the non-laboratory-based score developed using the National Health and Nutrition Examination Survey (NHANES) I population data in the USA was used to calculate total CVD risk. This equation was found to be highly correlated with commonly used laboratory scores in SA populations. ${ }^{[7]}$ The sociodemographic determinants associated with high-risk scores $(\geq 20 \%)$ were also ascertained.

\section{Methods}

\section{Study population and sampling procedure}

A sample of 25 - 74-year-old men and women in the predominantly black residential areas of Langa, Guguletu, Crossroads, Nyanga and Khayelitsha in Cape Town participated in this cross-sectional study in 2008/09. The sampling procedure for the current study included a three-stage cluster sampling and has been described in detail elsewhere. ${ }^{[8]}$ The prespecified age and gender quotas included disproportionate sampling across age groups to ensure at least 50 men and women in each gender category. Among other criteria individuals on tuberculosis or antiretroviral therapy or who had received cancer treatment within the past year were excluded. Additionally, participants with a self-reported history of ischaemic heart disease (IHD) or stroke were excluded for this analysis.

\section{Data collection}

Data collected by administered questionnaires included sociodemographic characteristics, medical history and self-reported tobacco use (World Health Organization (WHO) STEP-wise surveillance questionnaire). ${ }^{[9]}$ Assets defining wealth were recorded and included ownership of consumer items such as a radio, television, telephone, refrigerator, personal computer, washing machine, motor vehicle, bicycle and electricity, and the source of drinking water and toilet facilities.

Height and weight were measured using standardised techniques. Three BP measurements were taken at 2-minute intervals and the average of the second and third measurements was used in the analyses.

Blood samples for lipid and glucose estimations were drawn following an overnight fast of 10 hours. A standard oral glucose tolerance test was then administered and blood samples taken 120 minutes later. ${ }^{[10]}$ Blood samples were kept on ice and transported to the laboratory within 6 hours to be centrifuged, aliquoted and stored at $-80^{\circ}$ until the assays were performed.

\section{Definitions}

Diabetes was diagnosed according to the 1998 WHO criteria, ${ }^{[10}$ the use of hypoglycaemic agents or the subject having being told they were diabetic by a doctor/nurse. Hypertension was defined as $\mathrm{BP} \geq 140 / 90 \mathrm{mmHg}$ or using antihypertensive agents. BMI was computed as $\mathrm{kg} / \mathrm{m}^{2}$. Smoking status was defined as currently smoking daily or occasionally.

The absolute risk of having a CVD event, defined as IHD, stroke, transient ischaemic attack or heart failure, within 10 years was calculated using the Framingham ${ }^{[11]}$ and NHANES I equations. ${ }^{[12]} \mathrm{A}$ score $\geq 20 \%$ was considered to indicate high risk and $10-19.99 \%$ to indicate moderate risk.

\section{Statistical analysis}

Data analyses were done using STATA 12. Descriptive statistics, including crude prevalence, were calculated using the weights based on the sample design and adjusted for the realised sample. A principal component analysis of the pooled data, based on the assets that defined wealth, was used to develop an asset index ${ }^{[13]}$ and categories of relative wealth were created using tertiles. The first component of the principal component analysis placed the highest loading on having a toilet and a tap inside the house and explained $31.0 \%$ of the variation. The second component seemed to measure the ownership of luxury items such as a car and a personal computer and explained $12.2 \%$ of the variation. All variables, except bicycle and telephone, had approximately equal loadings.

The equations for calculating the 10 -year risk of developing a CVD event are gender-specific and include the variables of age, diabetes status, smoking status, treated and untreated systolic BP, TC and HDL cholesterol levels for the laboratory-based equations. BMI replaced lipids in the non-laboratory-based equations. ${ }^{[1,12]}$ Concordance correlation coefficients determined the correlations of the mean scores calculated by the laboratory-based equations with the two non-laboratory based equations. The McNemar test compared the frequencies of estimated high risk calculated using the laboratorybased equations with the two non-laboratory based equations.

The univariate analyses are presented as mean or percentage values with $95 \%$ confidence intervals (CIs). The unadjusted survey-based odds ratio (OR) and 95\% CI for the associations of the sociodemographic variables with total CVD risk $\geq 20 \%$ were calculated. On account of the high level of correlation between the risk scores, only the associations for the Framingham laboratorybased CVD risk scores are presented in men and women. Survey multiple logistic regression analyses determined the independent associations of the sociodemographic variables with estimated total CVD risk $\geq 20 \%$. The same sociodemographic variables were included in the unadjusted and adjusted analyses. $p$-values are presented for the adjusted analyses.

The University of Cape Town's Research and Ethics Committee approved the study. All participants signed informed consent.

\section{Results}

The realised study sample comprised 1099 participants, 1025 of whom (369 men and 656 women) were included in this analysis. Seventy-four participants with a self-reported history of IHD or stroke were excluded. The overall response rate was $86 \%$, the non-responders, i.e. the selected people who the study team were unsuccessful in contacting, totalling 187 (79 men).

The overall mean 10-year risk for a CVD event estimated using the laboratory-based scores was low at $7.1 \%$ and significantly higher in men than in women $(9.0 \%$ v. $5.4 \% ; p<0.001)$ (Table 1$)$. The non-laboratory-based mean scores were comparable to these laboratory-based estimates (Table 1 and Fig. 1). The concordance correlations between the Framingham laboratory and non-laboratory risk scores were very high at 0.936 for men and 0.924 for women. The concordance correlations between the Framingham laboratory-based 
Table 1. Total CVD risk estimates using laboratory- and non-laboratory-based equations in men and women

\begin{tabular}{|c|c|c|c|c|c|c|c|c|c|}
\hline & \multicolumn{3}{|c|}{$\operatorname{Men}(N=369)$} & \multicolumn{3}{|c|}{ Women $(N=656)$} & \multicolumn{3}{|c|}{ Total $(N=1025)$} \\
\hline & $n$ & $\%$ & $95 \% \mathrm{CI}$ & $n$ & $\%$ & $95 \% \mathrm{CI}$ & $n$ & $\%$ & $95 \% \mathrm{CI}$ \\
\hline \multicolumn{10}{|c|}{ Framingham CVD risk using lipid profiles } \\
\hline Mean & & 9.0 & $7.7-10.3$ & & 5.4 & $4.7-6.1$ & & 7.1 & $6.4-7.9$ \\
\hline Moderate risk (10 - 19.99\%) & 57 & 12.2 & $9.2-16.0$ & 71 & 8.3 & $6.3-10.9$ & 128 & 10.2 & $8.3-12.4$ \\
\hline High risk $(\geq 20 \%)^{*}$ & 67 & 13.0 & $9.9-17.0$ & 55 & 6.1 & $4.6-8.0$ & 122 & 9.4 & $7.6-11.6$ \\
\hline \multicolumn{10}{|l|}{ Framingham CVD risk using BMI } \\
\hline Mean & & 11.1 & $9.6-12.6$ & & 6.8 & $5.9-7.7$ & & 8.8 & $7.9-9.7$ \\
\hline Moderate risk (10 - 19.99\%) & 71 & 16.2 & $12.6-20.7$ & 83 & 10.3 & $8.2-12.8$ & 154 & 13.1 & $11.0-15.5$ \\
\hline High risk $(\geq 20 \%)^{*}$ & 87 & 17.4 & $13.8-21.8$ & 74 & 8.4 & $6.4-10.9$ & 161 & 12.7 & $10.6-15.2$ \\
\hline \multicolumn{10}{|l|}{ CVD risk using BMI (NHANES I) } \\
\hline Mean & & 9.0 & $7.6-10.3$ & & 8.7 & $7.6-9.8$ & & 8.8 & $7.9-9.8$ \\
\hline Moderate risk (10 - 19.99\%) & 64 & 14.5 & $10.9-19.0$ & 84 & 10.1 & $8.0-12.7$ & 148 & 12.2 & $10.1-14.6$ \\
\hline High risk $(\geq 20 \%)^{\dagger}$ & 65 & 12.2 & $9.3-15.7$ & 121 & 14.0 & $11.4-17.0$ & 186 & 13.1 & $11.1-15.5$ \\
\hline
\end{tabular}

and NHANES I risk scores were 0.950 and 0.786 in men and women, respectively.

The prevalence of estimated high total CVD risk $(\geq 20 \%)$ according to the laboratory-based scores was $13.0 \%$ in men and $6.1 \%$ in women $(p<0.001)$ (Table 1$)$. In men, the frequencies of estimated high risk were significantly different between the laboratory-based and the Framingham non-laboratory-based scores $(p<0.001)$ but were similar between the former and the NHANES I non-laboratory scores $(p=0.695)$. Taking the laboratory-based scores as the gold standard, the sensitivity was $97.0 \%$ and $79.1 \%$, respectively, and the specificity $92.7 \%$ and $96.0 \%$, respectively. In women, the frequencies of estimated high risk were significantly different for both comparisons $(p<0.001)$. The sensitivity of $98.2 \%$ and $100 \%$, respectively, was very high. The specificity for the comparison of estimated high risk between the two Framingham equations was $96.7 \%$ and that between the laboratory-based and the NHANES I nonlaboratory scores $89.0 \%$.

The prevalences of the individual CVD risk factors were similar in men and women for hypertension $(p=0.985)$, diabetes $(p=0.071)$ and HDL cholesterol:TC $<20 \%$ ( $p=0.170$ ) (Fig. 2). Men and women differed in smoking prevalence, which was significantly higher in men than in women (58.4\% v. 9.8\%; $p<0.001)$. Overweight and obesity was significantly higher in women $(82.0 \%)$ than in men $(29.2 \%)(p<0.001)$.

As shown in Table 2, the adjusted odds for estimated high CVD risk by the Framingham laboratory-based scores were significantly associated with $\leq 7$ years of education $(p<0.001)$

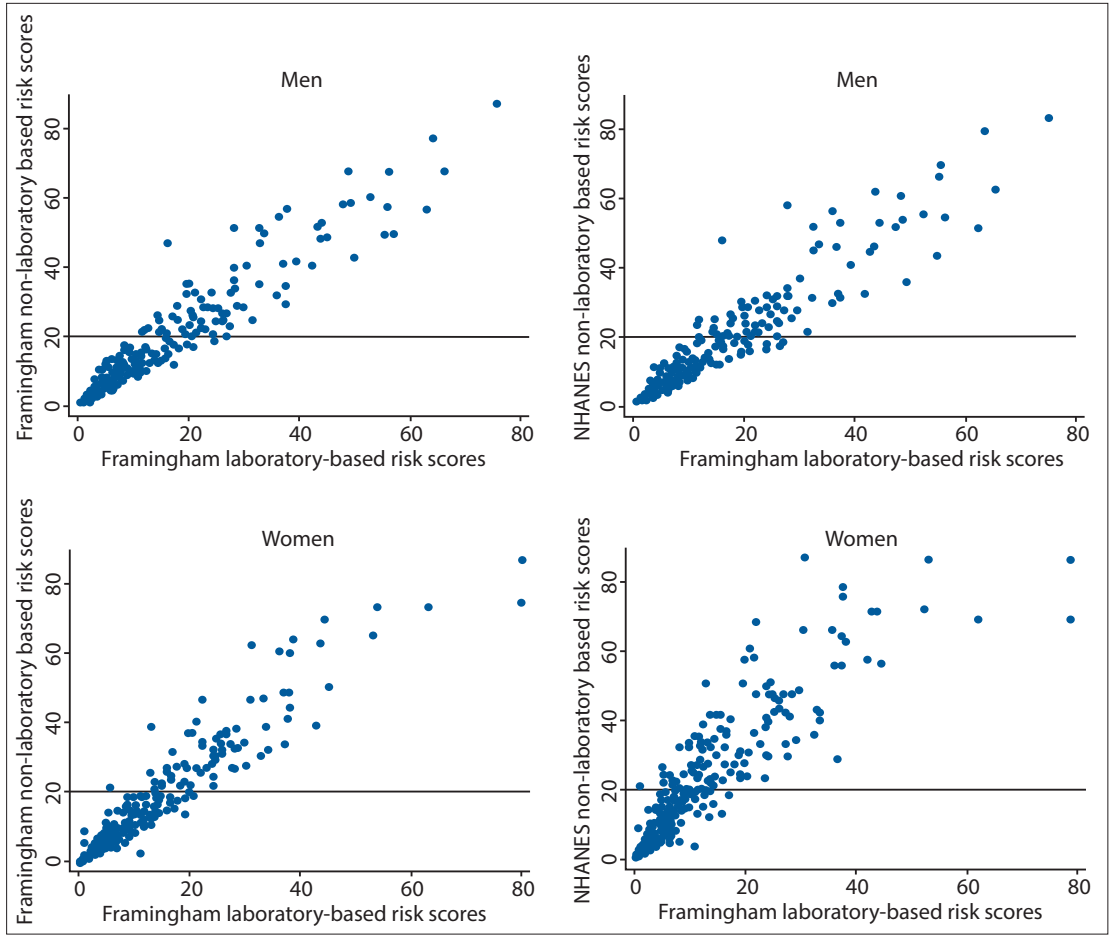

Fig. 1. Scatterplots of the Framingham laboratory-based total CVD risk scores with the Framingham and NHANES non-laboratory-based CVD risk scores $(C V D=$ cardiovascular disease; NHANES = National Health and Nutrition Examination Survey).

and unemployment $(p=0.021)$ in men. The significant adjusted odds for estimated high CVD risk by the laboratory-based scores in women were $\leq 7$ years of education $(p<0.001)$, better-quality housing $(p=0.044)$ and being in the poorest wealth tertile $(p=0.013)$. The work category with pensioners was associated with high CVD risk for both men and women because of the link with age, which is a function of the risk equation.
In the Framingham non-laboratory-based logistic models, compared with the laboratory-based analyses, there were no changes in the direction or significance of the variables except for housing, which was no longer significant in women $(p=0.231)$. In the NHANES I logistic models, unlike the Framingham laboratory-based analyses, better housing ( $p=0.044$ ) was associated with an estimated high CVD risk in men, while 
in women housing $(p=0.133)$ and wealth ( $p=0.094$ ) were no longer significant.

\section{Discussion}

The CRIBSA study, which is among the first to determine the 10-year risk of developing a CVD event in an urban African population in SA, found a modest prevalence of high risk $(\geq 20 \%)$ in men and a low to moderate prevalence in women. This suggests that for cost-effective management, a relatively smaller proportion of participants require treatment compared with those with prevalent individual risk factors. For example, while only $13.0 \%$ of men and $6.1 \%$ of women would require interventions according to the Framingham laboratory-based high-risk estimates, hypertension prevalence was high at $35 \%$. This is especially relevant in view of the potential burden that may be imposed on healthcare services if all individuals with only high BP were treated with medication. Basing treatment decisions on a total CVD risk assessment approach enables individuals who would benefit the most from treatment to be identified and also results in optimal and cost-effective management, as shown by Gaziano et al. ${ }^{[14]}$ in an analysis of the SA hypertension guidelines.

The data confirm that the use of nonlaboratory scores in clinical practice may be a feasible alternative in our setting. There was a high correlation between the mean laboratory-based and non-laboratorybased scores, and a high proportion of participants identified as at high risk on the laboratory-based estimates were also identified by the non-laboratory estimates. Gaziano et al. ${ }^{[7]}$ also reported high correlations between laboratory- and non- laboratory-based scores in SA populations. Eliminating the need for costly laboratory measurements will enhance accessibility and utility of the total CVD risk assessment tool $^{[4]}$ and is particularly relevant in lowresource settings such as SA that face a high burden of multiple diseases.

Estimated high CVD risk by Framingham laboratory- and non-laboratory-based scores was found to be significantly related to unemployment in men, poverty in women and lower education levels in both. These are vulnerable groups that are likely to be less aware of the risks of CVD and may have difficulty in accessing healthcare because of financial constraints. There is therefore a pressing need to increase awareness of CVD among these individuals. Additionally, they should be targeted for screening and prevention of CVD and its risk factors, and their access to healthcare, if compromised, should be facilitated.

In view of the association of high CVD risk with poverty or lower socioeconomic status as defined by education level and asset index or wealth tertiles, the relation between better-quality housing and estimated high risk by the Framingham laboratory-based scores in women and the NHANES I scores in men was unexpected. That wealth, education and housing quality may not be well correlated possibly accounts for these findings, underscoring the fact that socioeconomic dynamics are complex and further research in this area is needed.

Despite the high prevalence of CVD risk factors in both men and women, the two-fold greater predicted high risk of Framinghambased CVD in men compared with women is a function of the equations, which allocate

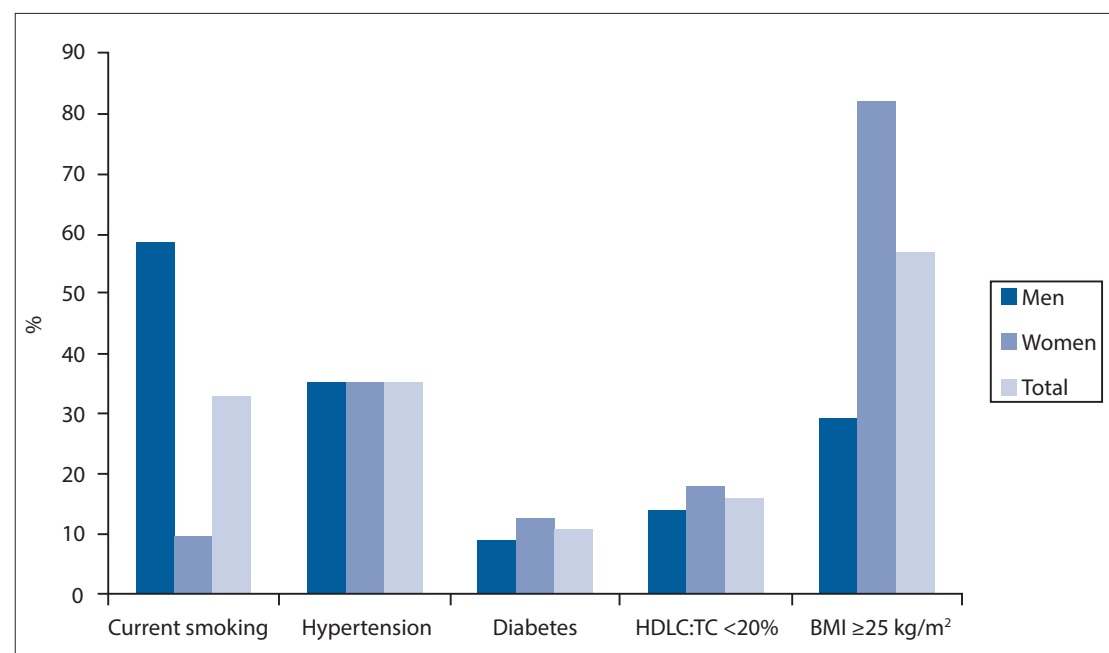

Fig. 2. Prevalence of cardiovascular disease risk score components presented according to gender. (Hypertension $=$ blood pressure $\geq 140 / 90 \mathrm{mmHg}$ or on hypertension treatment; diabetes $=$ raised fasting glucose $\geq 7.0 \mathrm{mmol} / \mathrm{l}, 2$-hour glucose $\geq 11.1 \mathrm{mmol} / \mathrm{l}$ or known diabetes; HDLC = high-density lipoprotein cholesterol; $T C=$ total cholesterol; $B M I=$ body mass index.) greater weight to men. In women, while high scores identify those at high risk, lower scores do not sufficiently ensure that individual women are at low risk. It is difficult for women aged $<75$ years, even with several markedly elevated risk factors, to be classified as at high risk. ${ }^{[15]}$ Factors beyond the risk scores therefore need to be considered when determining therapy in women. These models do not incorporate all CVD risk factors, particularly physical activity and stress. They also do not consider the duration of exposure to a risk factor, or include relevant family history. ${ }^{[15]}$ In addition, for CVD risk management in women, medical and lifestyle history, markers of preclinical disease and other conditions need to be considered when determining the intensity of preventive therapy. Recommendations also include a lower cut-point for defining high risk in women as $\geq 10 \% 10$-year risk for all CVD compared with the traditionally $\geq 20 \%$ 10-year risk estimate utilised. ${ }^{[15]}$ The prevalence of high risk in women would then be $14.4 \%, 18.7 \%$ and $24.1 \%$ by the laboratory-based and non-laboratorybased Framingham and NHANES 1 scores, respectively.

Nevertheless, while there are some recognised limitations, 10-year risk estimations represent an improvement over clinical judgement alone for appropriate risk stratification. Considering that a systematic approach to total CVD risk estimation seems to result in better risk factor control, ${ }^{[4]}$ the weaknesses of the CVD risk prediction models should not defer the control of the major risk factors through this cost-effective approach. ${ }^{[2]}$ Total CVD risk assessment is likely to remain an important therapeutic component for individuals and populations.

\section{Study limitations}

Limitations of the study included the low sample realisation in men (64\%), which necessitated higher sampling weights and a loss of precision. The application in this study of risk estimation tools developed primarily in populations of European origin in wealthy regions with different incidences of CVD events to our population is another limitation.

\section{Conclusion}

Despite the high prevalence of many individual CVD risk factors, the estimates of a $\geq 20 \% 10$-year risk for a CVD event in the black population of Cape Town were moderate in men and low to moderate in women, depending on the equation used. This implies that for optimal cost-effective 


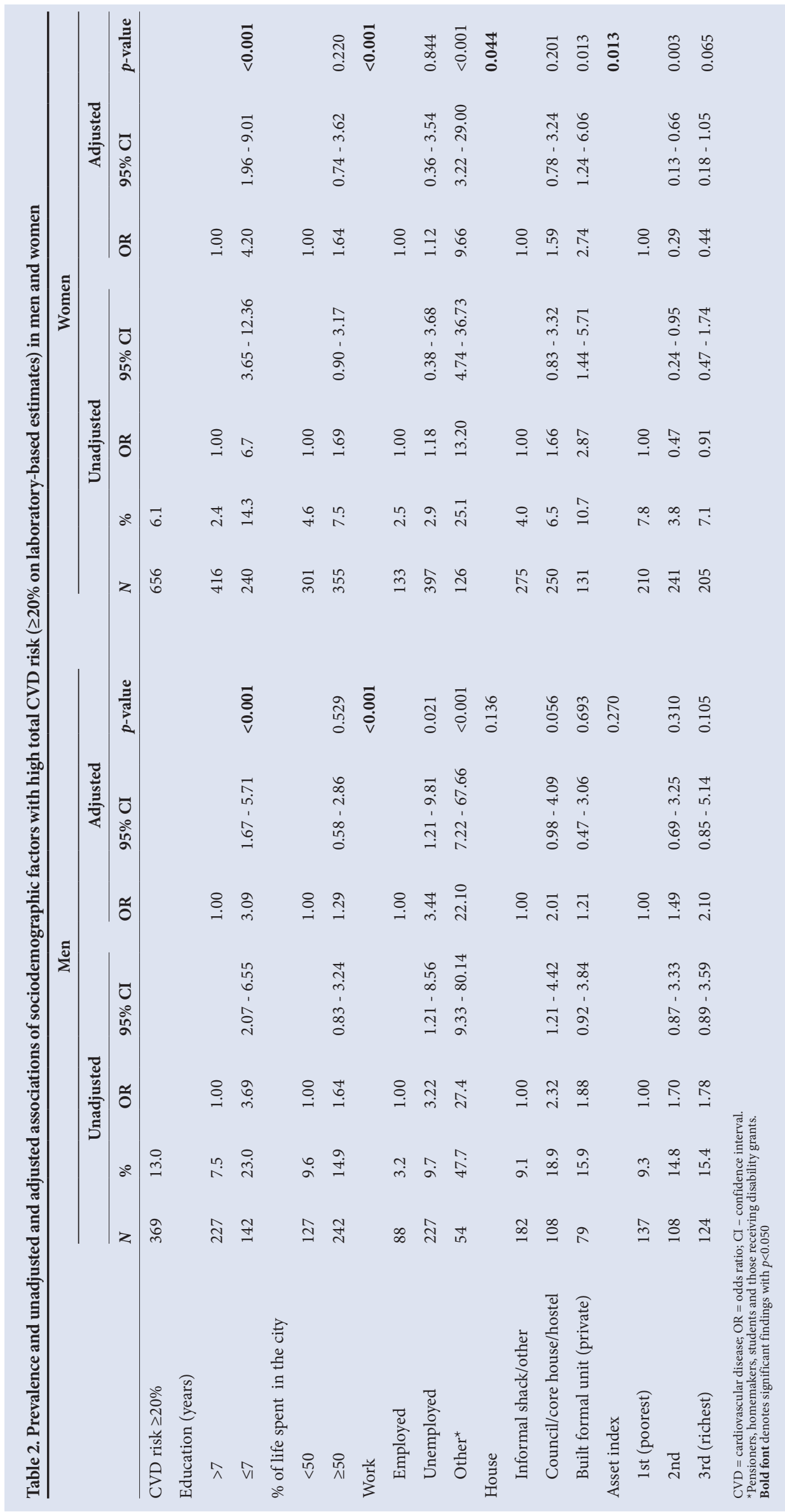

management of CVD in this population, fewer individuals require medical intervention compared with those with raised single risk factors. The comparability of the Framingham laboratory- and non-laboratorybased CVD risk estimates illustrates the utility of the latter in this resource-limited setting and could further optimise costeffective strategies. In view of the fact that in this study high CVD risk was associated with unemployed men, the poorest women and less-educated adults, CVD management needs to target these vulnerable groups. Future research will be required to determine whether the total CVD risk approach results in improved health outcomes and benefits for the healthcare system in SA.

Acknowledgements. We thank the participants, fieldworkers, Medical Research Council research nurse fieldworkers Debbie Jonathan and Theresa Gogela, fieldwork co-ordinator Erica April, study manager Serena van Haght, statisticians Nomonde Gwebushe, Rebecca Shanmugam and Ria Laubscher, and Dr Kirsty Bobrow. We thank the City of Cape Town for provision of the aerial maps.

Conflict of interest. NL has received honoraria from Novartis for serving on the steering committee for the Navigator Trial and travel support from Novo Nordisk, Eli Lilly Laboratories and Sanofi Aventis. All other authors report no potential conflicts of interest, including specific financial interests, relevant to the subject of this manuscript. Funding. This work was supported by an unrestricted grant from Servier Laboratories (South Africa), the Medical Research Council of South Africa, the Initiative for Cardiovascular Health Research in Developing Countries (IC Health) Foundation Council, and Brigham and Women's Hospital, Harvard University. The funders had no role in study design, data collection and analysis, decision to publish, or preparation of the manuscript. 


\section{References}

1. Ker JA, Oosthuizen H, Rheeder P. Decision-making using absolute cardiovascular risk reduction and incremental cost-effectiveness ratios: A case study. Cardiovasc J Afr 2008;19(2):97-101,

2. Mendis S. The contribution of the Framingham Heart Study to the prevention of cardiovascular disease: A global perspective. Prog Cardiovasc Dis 2010;53(1):10-14. [http://dx.doi.org/10.1016/3. pcad.2010.01.001]

3. Bitton A, Gaziano TA. The Framingham Heart Study's impact on global risk assessment. Prog Cardiovasc Dis 2010;53(1):68-78. [http://dx.doi.org/10.1016/j.pcad.2010.04.001]

4. Cooney MT, Dudina A, D'Agostino R, Graham IM. Cardiovascular risk-estimation systems in primary prevention: Do they differ? Do they make a difference? Can we see the future? Circulatio 2010;122(3):300-310. [http://dx.doi.org/10.1161/CIRCULATIONAHA.109.852756]

5. Klug E. South African dyslipidaemia guideline consensus statement. S Afr Med J 2012:102(3):178-187. 6. Seedat YK, Croasdale MA, Milne FJ, et al. South African hypertension guideline 2006. S Afr Med J 2006;96(4):337-362

7. Gaziano TA, Pandya A, Steyn K, et al. Comparative assessment of absolute cardiovascular disease risk characterization from non-laboratory-based risk assessment in South African populations. BMC Med 2013;11(1):1-11. [http://dx.doi.org/10.1186/1741-7015-11-170]

8. Peer N, Steyn K, Lombard C, Lambert EV, Vythilingum B, Levitt NS. Rising diabetes prevalence among urbandwelling black South Africans. PloS One 2012;7(9):e43336. [http://dx.doi.org/10.1371/journal.pone.0043336]

9. Bonita R deCourten M Dwyer T Jamrozik K, Winkelmann R. Surveillance of Risk Factors for Noncommunicable Diseases: The WHO STEPwise approach. Geneva: World Health Organization, 2001.
10. World Health Organization. Definition, Diagnosis and Classification of Diabetes Mellitus and its Complications: Report of a WHO Consultation. Geneva: World Health Organization, 1999.

11. D’Agostino RB Sr, Vasan RS, Pencina MJ, et al. General cardiovascular risk profile for use in primary care: The Framingham Heart Study. Circulation 2008;117(6):743-753. [http://dx.doi.org/10.1161/ CIRCULATIONAHA.107.699579]

12. Gaziano TA, Young CR, Fitzmaurice G, Atwood S, Gaziano JM. Laboratory-based versus nonlaboratory-based method for assessment of cardiovascular disease risk: The NHANES I Follow-up Study cohort. Lancet 2008;371(9616):923-931. [http://dx.doi.org/10.1016/S0140-6736(08)60418-3]

13. Filmer D, Pritchett LH. Estimating wealth effects without expenditure data - or tears: An application to educational enrollments in states of India. Demography 2001;38(1):115-132.

14. Gaziano TA, Steyn K, Cohen DJ, Weinstein MC, Opie LH. Cost-effectiveness analysis of hypertension guidelines in South Africa: Absolute risk versus blood pressure level. Circulation 2005:112(23):35693576. [http://dx.doi.org/10.1161/CIRCULATIONAHA.105.535922]

15. Mosca L, Benjamin EJ, Berra K, et al. Effectiveness-based guidelines for the prevention of cardiovascular disease in women - 2011 update: A guideline from the American Heart Association. Circulation 2011;123(11):1243-1262. [http://dx.doi.org/10.1161/CIR.0b013e31820faaf8]

Accepted 2 June 2014 